Revista Universo Contábil, ISSN 1809-3337

FURB, v. 5, n.3, p. 43-59, jul./set., 2009

doi:10.4270/ruc.2009321

Disponível em www.furb.br/universocontabil

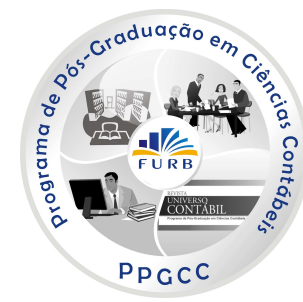

\title{
FORMULAÇÃO DE CARTEIRAS HIPOTÉTICAS DE ATIVOS FINANCEIROS USANDO A TÉCNICA MULTIVARIADA DE ANÁLISE DE AGRUPAMENTO'
}

\section{HYPOTHETICAL PORTFOLIO FORMULARIZATION OF FINANCIAL ASSETS USING THE TECHNIQUE MULTIVARIATE ANALYSIS OF CLUSTERS}

\begin{abstract}
Wesley Vieira da Silva
Doutor em Engenharia de Produção pela EPS/UFSC Professor do Programa de Pós-Graduação em Administração Estratégica da PUC/PR Endereço: Rua Imaculada, 1155, Bloco Acadêmico, $2^{\circ}$ andar - Campus Curitiba, Bairro Prado Velho CEP: 80215-901 - Curitiba/PR - Brasil E-mail: wesley.vieira@pucpr.br Telefone: (41) 3271-1476

Aline Gonçalves Lins

Mestranda em Administração Estratégica na Pontifícia Universidade Católica do Paraná Endereço: Rua Imaculada, 1155, Bloco Acadêmico, $2^{\circ}$ andar - Campus Curitiba, Bairro Prado Velho CEP: 80215-901 - Curitiba/PR - Brasil E-mail: aline.lins@gvt.com.br

Telefone: (41) 3271-1476

Liliane Gomes

Mestranda em Administração Estratégica na Pontifícia Universidade Católica do Paraná Endereço: Rua Imaculada, 1155, Bloco Acadêmico, $2^{\circ}$ andar - Campus Curitiba, Bairro Prado Velho CEP: 80215-901 - Curitiba/PR - Brasil

E-mail: gomesliliane@yahoo.com.br

Telefone: (41) 3271-1476

Sandro Marques

Mestre em Administração Estratégica pela Pontifícia Universidade Católica do Paraná Endereço: Rua Imaculada, 1155, Bloco Acadêmico, $2^{\circ}$ andar - Campus Curitiba, Bairro Prado Velho CEP: 80215-901 - Curitiba/PR - Brasil E-mail: smarques@peer.com.br Telefone: (41) 3271-1476
\end{abstract}

\footnotetext{
1 Artigo recebido em 14.10.2007. Revisado por pares em 02.06.2008. Reformulado em 27.12.2008. Recomendado em 28.01.2009 por Ilse Maria Beuren (Editora). Publicado em 30.09.2009. Organização responsável pelo periódico: FURB.
} 


\section{RESUMO}

Este estudo tem por objetivo identificar grupos de ações com características similares (carteiras hipotéticas), visando à alocação de capital disponível para investimento de forma a conhecer o risco potencial e o retorno possível para um potencial investidor. Para tanto, utiliza-se a técnica de análise de agrupamentos (cluster analysis), a partir das ações negociadas na Bolsa de Valores de São Paulo. O período analisado refere-se ao quadriênio de 1998 a 2002, para a construção dos clusters, e o período compreendido entre 2002 e 2006 foi usado para avaliar o desempenho das carteiras formadas com base nessa análise de agrupamento. Os resultados auferidos identificaram cinco carteiras hipotéticas, sendo três delas consideradas ideais para um potencial investimento, a saber: carteira 1 - com baixo risco e baixo retorno; carteira 3 - com baixo risco e alto retorno e carteira 5 - com alto risco e alto retorno. A avaliação do comportamento das carteiras hipotéticas sugeridas apresentou desempenho dentro do esperado no quadriênio 2002 a 2006, comprovando a adequação da técnica de análise de clusters. Contudo, apesar dos resultados observados, ressalta-se que uma análise conjuntural aliada aos estudos matemáticos e (ou) econométricos é indispensável à construção de boas carteiras de investimento.

Palavras-chave: Carteiras. Investimentos. Agrupamento. Finanças.

\section{ABSTRACT}

The objective this paper is to identify groups of stocks with similar characteristics (hypothetical portfolio), having as a goal the allocation of capital available for investment so as to understand the potential risks and possible returns that a potential investor could have. Therefore, cluster analysis technique was used on stocks traded at the São Paulo Stock Exchange. The period analyzed was from 1998 to 2002 for the construction of the clusters, and from 2002 to 2006 for the evaluation of the behavior of the portfolios originated from the cluster analysis. Five hypothetical portfolios were identified, being three of them considered suitable for a potential investor, as follows: Portfolio 1 - low risk, low return; Portfolio 3 low risk, high return; and Portfolio 5: high risk, high return. The behavior evaluation of the suggested hypothetical portfolios showed the expected performance within the period from 2002 through 2006, proving the adequation of the cluster analysis technique. Nevertheless, despite the observed results, it is emphasized that a conjunctural analysis associated with mathematical and (or) econometric studies is essential to the construction of good investment portfolios.

Keywords: Portfolios. Investments. Clusters. Finances.

\section{INTRODUÇÃO}

Um dos principais objetivos dos administradores de carteiras é a forma de como deve ser avaliado um conjunto de ativos sob regime de incerteza. Na verdade, o que eles querem é garantir que a sua carteira corra o mínimo de risco possível e obtenha rendimentos esperados acima daquele oferecido pelo mercado.

Os estudos e modelos que procuram encontrar estratégias de investimento em ações, de modo a formar carteiras capazes de apresentar retornos superiores aos índices de mercado com riscos dentro de um padrão tolerável, são tão antigos quanto as próprias Bolsas de Valores. Assim, originou-se o conceito de carteira de investimentos como um grupo de ativos 
que pertence a um investidor, pessoa física ou jurídica que busca a maximização da relação entre risco incorrido e retorno esperado.

Mesmo que reduzido, o risco é uma variável que se encontra presente em todos os investimentos no mercado acionário. O investidor que concentra seus recursos em um único ativo está se expondo a um risco maior, uma vez que o capital alocado fica condicionado às oscilações de mercado e, assim, desprotegido de variações não previstas na economia.

O objetivo deste trabalho é construir carteiras hipotéticas de ações com características homogêneas em termos de risco e de retorno, pautando-se na técnica de análise de cluster, e utilizando-se de dados históricos, cujo período está compreendido entre 1998 e 2002. Para tanto, verificar-se-á, posteriormente, se o comportamento das mesmas carteiras foi mantido no período compreendido entre 2002 e 2006.

A justificativa para a realização deste trabalho reside na busca de uma métrica alternativa que auxilie um potencial investidor na formulação de grupos de ações em que possam alocar seu capital disponível para investimento de forma a conhecer o risco potencial e o retorno possível. Esse processo de tomada de decisão constitui a parte central de qualquer tipo de atividade econômica. Problemas de escolha são comuns em todas as áreas da economia, como, por exemplo, quanto produzir, que preço cobrar, o que comprar, como aplicar os recursos etc.

Um problema decisório só existe quando o responsável pela tomada de decisão pode escolher entre dois ou mais planos de ação alternativos. Além do mais, para que o problema decisório seja significativo, as ações alternativas devem ser distintas e separadas, não só no sentido em que representam escolhas diferentes, mas também no sentido em que implicam diferentes resultados; senão a escolha de alternativas pode representar muito pouco para a tomada de decisão.

$\mathrm{O}$ estudo organiza-se de forma a contextualizar o leitor com alguns dos fatores macroeconômicos que afetaram o mercado acionário brasileiro nos dois períodos estudados e com conceitos econométricos de alocação de recursos em carteiras. Após essa breve revisão, apresenta-se a metodologia utilizada para a realização da pesquisa, seguida pela apresentação dos resultados empíricos e das considerações finais.

\section{TEORIA DE CARTEIRAS}

Os estudos de Markowitz (1952) foram o marco inicial sobre os estudos de carteiras de investimento, introduzindo, dessa forma, a Teoria Moderna de Investimento (ou Teoria Moderna de Carteiras). Esses estudos apresentaram, pela primeira vez, um embasamento teórico matemático em relação à redução de risco resultante da diversificação (COSTA; ASSUNÇÃO, 2005).

Para Kritzman (2000), não há dúvida que o investidor procura maximizar a sua utilidade esperada, sendo assim, enfrenta a tarefa de compor uma carteira de ativos que maximize o retorno esperado versus o risco oferecido pelo total de ativos componentes dessa carteira.

Ribeiro e Ferreira (2005) acrescentam que o problema de composição de carteiras está intrinsecamente relacionado aos conceitos de risco e retorno. Segundo os autores, quando um operador do mercado financeiro ou administrador de empresas compõe uma carteira de ativos, o objetivo é basicamente obter o máximo de retorno possível, dado um nível aceitável de risco, ou obter o mínimo risco, fixando um nível de retorno.

Damodaran (2004) explica que os retornos esperados e variâncias são quase sempre estimados pelos retornos passados. O pressuposto implícito quando se utilizam variâncias históricas é de que distribuições passadas são boas indicadoras de distribuições futuras. Quando esse pressuposto é violado, como acontece quando características de ativos mudam significativamente com o tempo, as estimativas históricas podem não ser boas medidas de risco. 
A Teoria Moderna de Carteiras pode ser considerada uma ferramenta que permite ao investidor compor a carteira de investimento com diferentes ativos financeiros e otimizar a relação entre retorno e risco do investimento:

Essa teoria é desenvolvida seguindo a premissa de que os investidores avaliariam suas carteiras apenas com base no valor esperado e na variância das taxas de retorno no espaço de tempo considerado, sendo essas as duas únicas variáveis de decisão para a seleção dos ativos. Quando postos a escolher entre duas carteiras de mesmo risco, os investidores sempre escolheriam a de maior retorno e, da mesma forma, quando postos a escolher duas carteiras de mesmo retorno, sempre escolheriam a de menor risco (COSTA; ASSUNÇÃO, 2005, p. 17).

Esses princípios podem ser observados na Figura 1. Considerando $A, B$ e $C$ diferentes carteiras de investimentos, sendo $A$ e $C$ de mesmo risco e $A$ e $B$ de mesmo retorno esperado (sendo o retorno esperado e risco de $B$ e $C$ diferentes), o investidor sempre escolheria a carteira $A$ em relação à carteira $C$, pois $A$ possui um retorno esperado maior. Da mesma forma, a carteira $B$ sempre seria preferida em relação à carteira $A$, pois $B$ possui menor risco.

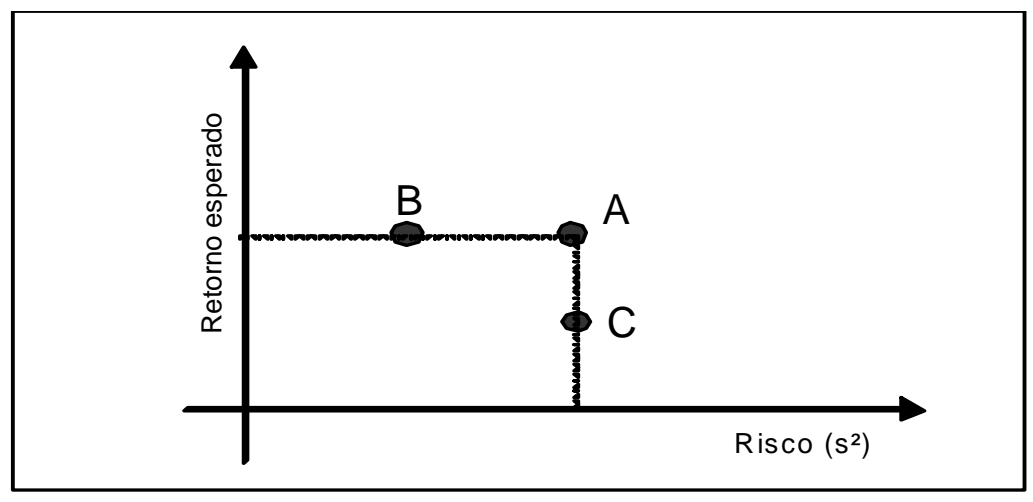

Figura 1 - Princípios de dominância entre ativos Fonte: adaptada de Vince (1999, p. 39).

Tomando-se como base os princípios de dominância acima descritos e considerandose um conjunto de ativos que podem ser combinados em diferentes proporções para a formação de carteiras de investimentos, é possível definir um conjunto limitado dessas carteiras de investimentos que são preferíveis em relação a todas as outras carteiras do conjunto. Essas carteiras, que são preferidas em relação às outras, são denominadas Carteiras Eficientes e o seu conjunto forma um conjunto denominado Fronteira Eficiente.

Os estudos de Markowitz (1952) liquidaram a concepção de que as estratégias ingênuas de diversificação, com a escolha aleatória dos títulos, trariam redução de risco na carteira. Para que uma carteira tenha riscos menores que os ativos que a compõem individualmente, é necessário que os preços dos ativos não variem em conjunto na mesma direção e proporção.

Para que um investidor possa auferir um maior retorno, ele dispõe do mecanismo de diversificação. Nesse ínterim, ele pode utilizar o recurso de distribuir os investimentos dentro de carteiras, que são grupos de ativos com o objetivo de maximizar a equação risco versus retorno. Assim, cabe ao gestor financeiro administrar a carteira de investimento de modo que se obtenha o maior retorno para um determinado nível de risco.

Markowitz (1952) propõe que os investidores selecionem as carteiras não com base no desempenho individual de ativos, mas levando em consideração o desempenho da carteira de forma agregada. A diversificação de carteiras pode levar à construção de carteiras "mais eficientes", em razão do efeito da correlação entre os ativos. 
Na visão de Dimson e Mussavian (1999), o trabalho de Markowitz mostrou que, desde que se disponha de um conjunto de dados e de capacidade computacional, é possível encontrar carteiras que forneçam o menor nível de risco para um pré-especificado nível de retorno do investimento.

Segundo Elton et al. (2004), um menor risco na carteira depende da possibilidade dos retornos dos ativos variarem em proporções diferentes ou que, enquanto alguns apresentem retornos positivos, outros apresentem retornos negativos. Assim, na determinação do risco de uma carteira, deve ser estimado um indicador que relacione os retornos dos títulos que compõem a carteira dois a dois. De acordo com Tosta de Sá (1999), esse indicador pode ser representado pelo coeficiente de correlação ou a covariância entre os títulos.

O coeficiente de correlação entre dois ativos é uma medida estatística que indica o grau de dependência linear entre as taxas de retorno desses ativos. Se os rendimentos positivos de um ativo estão associados aos rendimentos negativos do outro no mesmo período ou vice-versa, o coeficiente de correlação tem valor negativo. Se, por outro lado, os rendimentos positivos de um ativo estão associados aos rendimentos também positivos do outro ativo, ou negativos de um associado aos negativos do outro, no mesmo período, o coeficiente de correlação tem valor positivo. Caso sejam independentes, o coeficiente de correlação é igual à zero.

Esse coeficiente de correlação varia de +1 quando os resultados são perfeitamente correlacionados (sempre que um rendimento de um ativo é positivo, o do outro também é, assim como quando o rendimento de um é negativo, o do outro também é), até -1, quando a correlação é linear e perfeita no sentido inverso, ou seja, sempre que um é positivo, o outro é negativo e vice-versa.

Tosta de Sá (1999) também afirma que a covariância é outra medida estatística de dependência linear entre duas variáveis no caso das taxas de retorno de dois ativos. Da mesma forma que o coeficiente de correlação, quando as taxas de retorno não estão relacionadas linearmente, a covariância entre elas é zero. Para Tosta de Sá (1999, p. 70):

\footnotetext{
Quanto menor a covariância entre os retornos dos dois títulos, menor o risco da carteira e, mais, quando a covariância for negativa, o risco da carteira será menor do que a média ponderada do risco de cada um dos títulos que a compõem. Esse é o grande segredo da diversificação eficiente proposta por Markowitz.
}

Dessa forma, o risco de uma carteira pode ser reduzido se forem considerados ativos cuja covariância entre eles seja a menor possível. Elton et al. (2004) afirmam que se for considerado um número grande de ativos com retornos independentes, ou seja, com covariância igual a zero, a variância da carteira composta por esses ativos tenderá a zero. Apesar dessa possibilidade matemática, na prática, a maioria dos mercados possui correlação e covariância positivas entre as taxas de retorno de ativos. Elton et al. (2004, p. 71) mostram que "nesses mercados, não se consegue levar a zero o risco da carteira, mas ele pode ser muito inferior à variância do retorno de um ativo individualmente".

Com relação ao número ideal de ativos na carteira, Gitman e Joehnk (2005, p. 151) afirmam que "em média, a maior parte dos benefícios de diversificação, em termos de redução de risco, pode ser obtida formando-se carteiras que contenham de oito a 15 títulos selecionados aleatoriamente".

Elton, Gruber e Padberg (1977), num célebre artigo, mostraram como constituir a carteira ótima - proporções ótimas dos ativos - com e sem vendas a descoberto (short selling) se os agentes econômicos assumirem que existe um ativo sem risco e aceitarem as seguintes situações:

a) assumirem que o modelo single index descreve de uma forma adequada a estrutura da matriz variância/covariância; 
b) ou, alternativamente, assumirem que uma boa estimativa de todos os coeficientes de correlação é um único número, então um simples critério de decisão - que não envolve programação matemática - pode ser usado para chegar a uma solução ótima para o problema de construção de uma carteira.

Para Lintz e Renyi (1998), a análise do processo de diversificação de carteiras de investimentos é baseada em princípios lançados pelos trabalhos de Markowitz e Sharpe. Segundo Lintz e Renyi (1998), algumas premissas assumidas pelo modelo de Sharpe podem levar a imprecisões devido à forma de cálculo de alguns de seus parâmetros.

Segundo Francis (2001), para obter os melhores benefícios, o número ideal varia de 10 a 15 ativos diferentes nas carteiras. Acima desse limite, qualquer adição de título resulta em uma diversificação supérflua, pois poderia resultar nos seguintes problemas:

a) impossibilidade de boa gerência da carteira - em função da quantidade excessiva de ativos, o investidor não consegue acompanhar todos os ativos simultaneamente;

b) aquisição de ativos com baixo desempenho - uma quantidade excessiva de ativos pode levar à compra de algum ativo que não possua um retorno adequado ao seu nível de risco;

c) altos custos de pesquisa - quanto maior a quantidade de ativos, maior o esforço e o custo para análise e acompanhamento de cada ativo;

d) altos custos de corretagem - quanto maior o número de ativos, menor a quantidade alocada a cada um deles, o que pode resultar em maiores custos de corretagem.

Segundo Kritzman (2000), a identificação de uma carteira ótima passa pelo mapeamento da aversão ao risco dos investidores: fazendo variar os valores de aversão ao risco, é possível construir uma fronteira eficiente entre ações e carteiras. Para cada nível de tolerância, existe um grupo de ações que maximiza a utilidade esperada, que é a carteira ótima para aquele grau de tolerância ao risco.

Além disso, o aumento dos custos para formação e acompanhamento da carteira pode fazer com que o ganho líquido (retorno) do investidor seja reduzido, justificando, dessa forma, o limite máximo de 15 títulos para a formação de carteiras de investimentos mais eficientes.

Black e Litterman (1990) mostram, por sua vez, que o processo de otimização por meio da média/variância tende a atribuir um peso excessivo aos ativos com erros positivos nos rendimentos previsíveis esperados e atribuir pesos extremamente baixos aos outros ativos. Esses autores impuseram uma distribuição a priori aos rendimentos esperados, com base na hipótese de eficiência dos mercados, e usaram essa distribuição para derivar os rendimentos previstos $a$ posteriori de acordo com a modelagem bayesiana.

Em relação à aplicação da teoria de Markowitz no contexto internacional, cabe salientar que o trabalho de Herbert Grubel (1968) é considerado como um marco na área de finanças internacionais. Tal autor postula que a constituição de carteiras internacionais era suscetível de gerar ganhos de bem-estar diferentes dos ganhos de comércio e que os movimentos internacionais de capitais não são somente em função dos diferenciais de taxa de juro, mas também das taxas de crescimento dos asset holdings nos dois países. Assim, segundo o modelo delineado por esse autor, é possível que um acréscimo na taxa de crescimento de um dado país implique o aumento da aquisição de ativos estrangeiros, causando um fluxo de movimentos de capital, mesmo quando o diferencial das taxas de juro for zero.

Cooper e Kaplanis (1991) e French e Poterba (1991) mostram vantagens no processo de diversificação por meio de evidências empíricas ao diversificar carteiras em nível internacional.

\section{METODOLOGIA DA PESQUISA}

Dando continuidade ao presente estudo, esta seção traz os aspectos metodológicos 
propostos para a confecção da pesquisa. A subseção 3.1 trata do delineamento da pesquisa, a descrição da população e amostra está apresentada na subseção 3.2, enquanto a subseção 3.3 refere-se aos métodos estatísticos aplicados.

\subsection{Delineamento da pesquisa}

A presente pesquisa pode ser caracterizada como um trabalho de natureza descritiva, pois tem o objetivo de analisar o inter-relacionamento de ações negociadas na Bolsa de Valores de São Paulo a partir do estudo de suas características. Segundo Gil (2002), a pesquisa descritiva estabelece relações entre variáveis a partir da descrição das características de determinado fenômeno ou população.

Trata-se de uma pesquisa longitudinal, uma vez que envolve medições repetidas de uma mesma amostra fixa da população ao longo do tempo. Sendo assim, é possível analisar mudanças ocorridas nas variáveis, durante determinado intervalo de tempo (MALHOTRA, 2004).

Quanto aos procedimentos técnicos utilizados, o delineamento desta pesquisa é não experimental ou ex-post facto, não sendo possível manipular os dados e não havendo controle sobre as variáveis estudadas, pois trata de fenômenos já ocorridos. Esse delineamento considera constatações exclusivamente correlacionais entre as variáveis estudadas (GIL, 2002), atendendo ao objetivo do trabalho que é o de encontrar, a partir do estabelecimento das inter-relações entre as características das ações negociadas na Bovespa, grupos homogêneos.

\subsection{População e amostra}

Os dados utilizados na pesquisa foram coletados junto ao banco de dados da empresa Economática Software para Investimentos Ltda. e correspondem à totalidade de ações negociadas na Bolsa de Valores de São Paulo (totalizando 1.031 títulos). Os períodos de coleta dos dados referem-se a 30/06/1998, 28/06/2002 e 30/06/2006, respectivamente.

Para a formação das carteiras hipotéticas sugeridas (clusters), foi analisado o período de valorização de 1998 a 2002, que corresponde, no cenário político, ao segundo mandato do Presidente Fernando Henrique Cardoso. O acompanhamento das carteiras formuladas foi feito no período quadrienal seguinte, compreendido entre os anos de 2002 a 2006, correspondendo ao mandato do Presente Luiz Ignácio Lula da Silva.

Para a formação da amostra do estudo, fomam retirados os títulos que possuíam menos de $0,05 \%$ de liquidez no mercado em 2002, seguindo o mesmo procedimento adotado por Cia, Guarita e Pedote (1999), em seu estudo, que abrangeu os períodos de 1990 a 1994 e de 1994 a 1998. Esse filtro excluiu 885 ações. As ações com informações faltantes no período também foram excluídas da análise (50 ações). Sendo assim, a amostra final ficou em 95 títulos, que representavam, em 2002, aproximadamente 55\% do volume de movimentação da Bovespa.

\subsection{Descrição das variáveis}

As variáveis integrantes do estudo estão demonstradas no Quadro 1 e foram propostas por Cia, Guarita e Pedote (1999) e estão subdivididas em grupos, segundo os critérios que avaliam: a) Retorno; b) Risco; c) Tamanho da empresa; d) Cheapness, que faz a relação entre o valor atribuído pelo mercado ao título e uma variável financeira da empresa; e) Estrutura de capital; e d) Setor. 


\begin{tabular}{|c|c|c|}
\hline VARIÁVEIS & SIGLA & DESCRIÇÃO \\
\hline Retorno & VALOR Z & \% Valorização da ação no período de 4 anos. \\
\hline \multirow{3}{*}{ Risco } & BETA & $\begin{array}{l}\text { Coeficiente de risco }=\text { o risco relativo de um título individual, } \\
\text { mensurado pela covariância entre os retornos do título com os } \\
\text { retornos da carteira de mercado. }\end{array}$ \\
\hline & VOLAT & $\begin{array}{l}\text { Volatilidade }=\text { trata-se do desvio-padrão não-condicional } \\
\text { estimado das taxas de retorno. Uma ação pouco volátil não } \\
\text { registra alterações bruscas em seus preços. Quanto menor a } \\
\text { volatilidade, melhor para o papel. }\end{array}$ \\
\hline & LIQUI & $\begin{array}{l}\text { Liquidez na Bolsa = mede a concentração do capital da empresa. } \\
\text { É o percentual do volume negociado do ativo sobre o volume } \\
\text { total do mercado. Quanto maior o número de ações da companhia } \\
\text { no mercado, mais líquido é o seu papel. }\end{array}$ \\
\hline Tamanho & PTRLQP & Patrimônio Líquido ( $\mathrm{R} \$$ milhões), padronizado: $z=\frac{x-\mu}{\sigma}$ \\
\hline Cheapness & PVPA & $\begin{array}{l}\text { Preço da Ação/Valor Patrimonial = Relação entre preço e valor } \\
\text { patrimonial por ação. Quanto maior o PVPA, mais o mercado } \\
\text { está disposto a pagar pelos papéis. }\end{array}$ \\
\hline $\begin{array}{l}\text { Estrutura de } \\
\text { Capital }\end{array}$ & EXGATP & Exigível total/Ativo total, padronizado: $z=\frac{x-\mu}{\sigma}$ \\
\hline Setor & SETOR & Setor de atuação da empresa emissora. \\
\hline
\end{tabular}

Quadro 1 - Variáveis componentes da pesquisa

\subsection{Método de análise}

A técnica de análise de agrupamento tem como objetivo a busca por meio do agrupamento dos elementos de dados, baseando-se na similaridade entre eles, em que os grupos são determinados de forma a se obter homogeneidade dentro dos grupos e heterogeneidade entre eles.

Os dados foram tratados por meio da estatística multivariada de Análise de Agrupamento (cluster analysis), proporcionando os meios para investigar matematicamente as informações e objetivando agrupar as ações da Bovespa em carteiras hipotéticas de acordo com seus perfis de risco e retorno.

O método de análise de conglomerados ou cluster analysis é um conjunto de técnicas que se propõe a descobrir as inter-relações existentes entre as variáveis e, por meio disso, encontrar classificações ou tipologias que agrupem os dados (DILLON; GOLDSTEIN, 1984). Ao contrário da maioria das técnicas estatísticas, não faz distinção entre variáveis dependentes e independentes, e sim examina as relações de interdependência entre todo o conjunto de variáveis, separando-o em grupos similares (MALHOTRA, 2004).

A maior parte dos métodos de aglomeração consiste de processos relativamente simples, que não seguem pressupostos estatísticos rigorosos, enquanto o cluster analysis é uma heurística, baseada em algoritmos, portanto, facilmente aplicada a conjuntos de dados diversos (MALHOTRA, 2004).

Barroso e Artes (2003) explicam que a técnica de análise de agrupamentos tem aplicação em diversas áreas do conhecimento, uma vez que ajudam a identificar padrões de comportamento em bancos de dados por meio da formação de grupos homogêneos de casos.

Malhotra (2004) descreve que o processo de cluster analysis passa, obrigatoriamente, por uma série de etapas necessárias ao desenvolvimento do processo aglomerativo: a) formulação do problema; b) escolher uma medida de distância; c) escolher o algoritmo de aglomeração; d) decidir quanto ao número de conglomerados; e) interpretar e perfilar os agrupamentos; e f) avaliar o processo.

Um aspecto considerado importante para o uso dessa métrica refere-se à utilização do critério que mede a similaridade ou dissimilaridade de um conjunto. Nesse caso, deve-se 
buscar um critério que mensure a distância entre dois objetos ou que quantifique o grau de semelhança. Tal medida é conhecida na literatura estatística como coeficiente de parecença.

A medida de distância escolhida é a euclidiana, por ser a mais comumente utilizada por pesquisadores que lidam com esse método. Tal distância pode ser utilizada como um descritor dos objetos que investigarão os assemelhados, sendo indicada e conhecida para mensurar a proximidade entre os objetos.

Dentre os diversos processos de aglomeração, optou-se pela escolha do algoritmo de métodos hierárquicos aglomerativos com regra de Ward, que trabalham a partir da matriz de parecença. O método hierárquico de cluster consiste em uma série de sucessivos agrupamentos ou sucessivas divisões de elementos, em que os elementos são agregados ou desagregados. Nesse método de variância, cada objeto tem início em um conglomerado separado. Formam-se os conglomerados agrupando-se os objetos em conglomerados cada vez maiores.

Cabe salientar que, no método de variância, os conglomerados são gerados de modo a minimizar a variância dentro do conglomerado. No método de Ward, o objetivo é minimizar o quadrado da distância euclidiana em relação às médias dos conglomerados. Esse é o método mais utilizado em estudos de cluster na atualidade. Os grupos, nos métodos hierárquicos, são geralmente representados por um diagrama bi-dimensional, chamado de dendograma ou diagrama de árvore.

Desse modo, é possível minimizar a heterogeneidade interna (BARROSO; ARTES, 2003), ou seja, agrupar os indivíduos segundo as suas semelhanças, minimizando as diferenças dentro dos clusters. Essa ferramenta permite a definição automática do número de clusters.

A etapa de formulação do problema é descrita por Malhotra (2004) como a mais importante para o pesquisador, pois, a partir da seleção das variáveis sobre as quais se baseia o processo de aglomeração, é realizada a análise descritiva e de correlação entre tais variáveis.

A medida de correlação utilizada foi o coeficiente de correlação de Pearson, $r$, que é calculado de forma a medir a força entre duas variáveis numéricas e é utilizado para determinar se existe evidência estatística significativa da associação entre duas variáveis (LEVINE et al., 2005). É um índice que varia de -1 a +1 e quanto mais perto de $|1|$ maior é o grau de associação linear entre as variáveis tomadas par a par.

\section{APRESENTAÇÃO E ANÁLISE DOS DADOS}

Esta seção traz a apresentação e análise dos dados desta pesquisa empírica, que foi realizada com o objetivo de construir carteiras homogêneas de ativos com características similares por meio da técnica de cluster analysis, valendo-se de informações coletadas no período de 1998 a 2002 e verificando-se, a posteriori, se tais características se mantiveram no período de 2002 a 2006.

\subsection{Seleção das variáveis e tratamento dos dados}

A primeira etapa do tratamento dos dados consistiu na análise das estatísticas descritivas para a detecção de outliers, uma vez que estes podem interferir na constituição dos clusters. A Tabela 1 apresenta os índices básicos das sete variáveis quantitativas estudadas e possibilita verificar indícios de existência ou não de dados discrepantes nas variáveis. 
Tabela 1 - Estatísticas descritivas das variáveis em estudo

\begin{tabular}{c|ccccccc}
\hline Estatística & VALORZ & BETA & VOLAT & LIQUI & PTRLQP & PVPA & EXGATP \\
\hline $\mathrm{N}^{\circ}$. de observações & 95 & 95 & 95 & 95 & 95 & 95 & 95 \\
Mínimo & $-0,98$ & 0,00 & 0,00 & 0,00 & $-0,48$ & 0,00 & $-2,31$ \\
Máximo & 9,42 & 3,70 & 1,40 & 7,52 & 5,77 & 156,23 & 1,58 \\
Mediana & 1,07 & 0,70 & 0,40 & 0,12 & $-0,35$ & 2,09 & 0,04 \\
Média & 1,59 & 0,73 & 0,48 & 0,57 & 0,00 & 7,64 & 0,00 \\
Desvio padrão (n) & 2,09 & 0,41 & 0,23 & 1,13 & 0,99 & 17,77 & 0,99 \\
\hline
\end{tabular}

Os gráficos Box-plot, dispostos na Figura 2, possibilitam a detecção de outliers em seis das sete variáveis estudadas. Por meio dessa análise, são excluídos quatro títulos que estavam causando algum tipo de distorções na construção dos clusters.

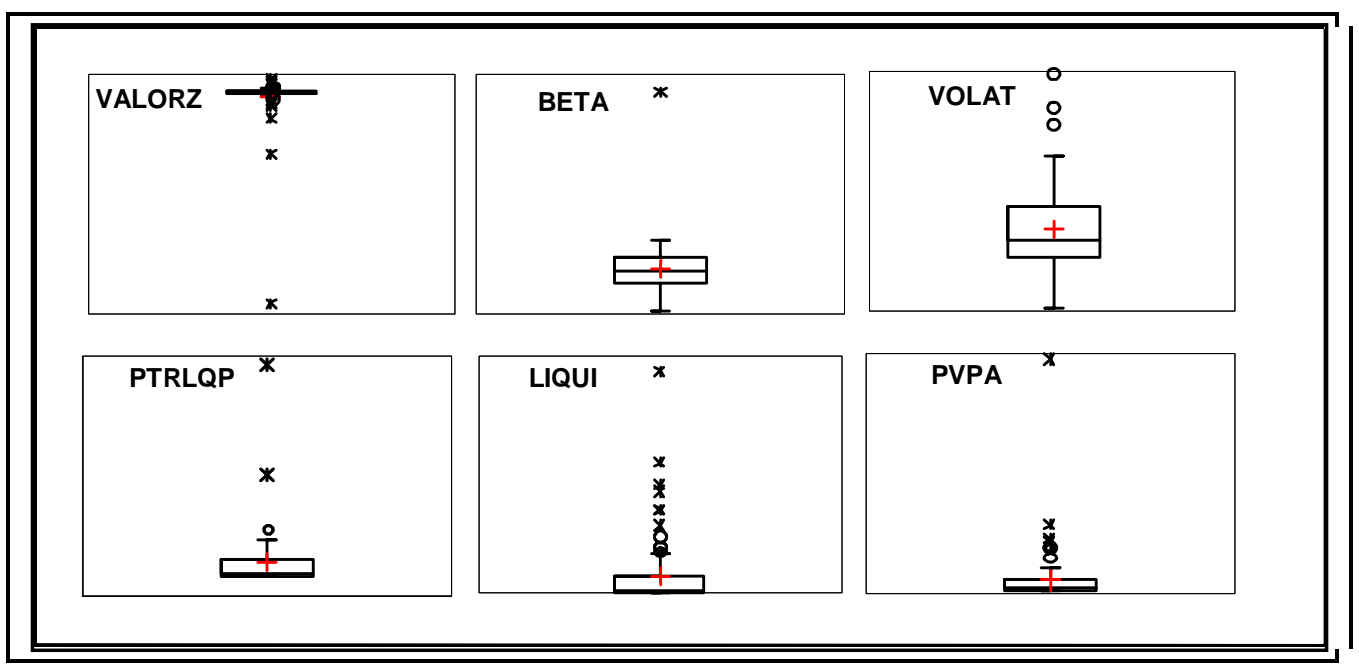

Figura 2 - Box Plot das variáveis estudadas

$\mathrm{Na}$ Tabela 2, apresentam-se as médias aritméticas de cada uma das variáveis pesquisadas para os quatro outliers que foram identificados, bem como suas respectivas empresas emissoras.

Tabela 2 - Observações outliers identificadas

\begin{tabular}{c|c|c|c|c|c|c|c|c}
\hline & AÇÕES & VALORZ & BETA & VOLAT & LIQUI & PTRLQP & PVPA & EXGATP \\
\hline & Inepar & & & & & & & \\
INEP4 & Construções & $-0,98$ & 0,08 & 0,09 & 0,31 & 0,90 & $-0,46$ & 1,16 \\
LIPR3 & LigntPar & 2,54 & 3,70 & 0,00 & 0,00 & 1,10 & $-0,47$ & $-0,46$ \\
BDLL4 & Bardella & $-0,26$ & 0,40 & 0,02 & 1,56 & 0,20 & $-0,45$ & $-1,12$ \\
CPFL4 & Ferro Ligas & $-0,84$ & 0,90 & 0,00 & 0,15 & 0,10 & $-0,46$ & $-0,93$ \\
\hline
\end{tabular}

A seleção das variáveis que irão formar os agrupamentos é realizada por meio da estimativa dos coeficientes de correlação linear de Pearson, buscando eliminar qualquer viés originado pela interdependência entre as variáveis que possa mascarar as inter-relações entre as observações (títulos).

Objetivando melhorar o desempenho da técnica de análise de agrupamentos, as variáveis cujos coeficientes de correlação apresentaram-se elevados e significantes foram eliminadas da etapa de construção dos conglomerados. Dessa forma, conforme demonstrado na Tabela 3, reduziu-se o número de dimensões utilizadas, passando-se a considerar as 
características VALORZ (família Retorno), BETA, LIQUI (família Risco) e PVPA (família Cheapness) na construção dos clusters.

Tabela 3 - Análise de correlação entre as variáveis do estudo

\begin{tabular}{c|c|c|c|c|c|c|c}
\hline Variáveis & VALORZ & BETA & VOLAT & LIQUI & PTRLQP & PVPA & EXGATP \\
\hline VALORZ & $\mathbf{1}$ & $-0,06$ & $\mathbf{- 0 , 3 0}$ & 0,08 & $-0,12$ & $-0,15$ & $\mathbf{- 0 , 2 5}$ \\
BETA & $-0,06$ & $\mathbf{1}$ & $\mathbf{0 , 3 1}$ & 0,19 & 0,17 & $-0,11$ & 0,11 \\
VOLAT & $\mathbf{- 0 , 3 0}$ & $\mathbf{0 , 3 1}$ & $\mathbf{1}$ & $-0,04$ & 0,03 & $-0,08$ & $\mathbf{0 , 3 3}$ \\
LIQUI & 0,08 & 0,19 & $-0,04$ & $\mathbf{1}$ & $\mathbf{0 , 5 6}$ & $-0,04$ & 0,06 \\
PTRLQP & $-0,12$ & 0,17 & 0,03 & $\mathbf{0 , 5 6}$ & $\mathbf{1}$ & $-0,03$ & $-0,15$ \\
PVPA & $-0,15$ & $-0,11$ & $-0,08$ & $-0,04$ & $-0,03$ & $\mathbf{1}$ & $-0,17$ \\
EXGATP & $\mathbf{- 0 , 2 5}$ & 0,11 & $\mathbf{0 , 3 3}$ & 0,06 & $-0,15$ & $-0,17$ & $\mathbf{1}$ \\
\hline
\end{tabular}

Nota: Os valores em negrito são significativamente diferentes de 0 , com um nível de significância alfa $=0,05$.

\subsection{Construção dos agrupamentos}

Utilizando-se as variáveis previamente selecionadas e buscando-se encontrar grupos com características homogêneas, realizou-se a análise de clusters, valendo-se do algoritmo do método hierárquico aglomerativo e da regra de definição da matriz de parecença pelo método de Ward. O método encontrou cinco grupos distintos. A Tabela 4 mostra a distribuição da quantidade de ações em cada grupo e a variância entre os objetos, calculada nos clusters.

Tabela 4 - Descrição dos grupos formados

\begin{tabular}{c|ccccc}
\hline Grupos & $\mathbf{1}$ & $\mathbf{2}$ & $\mathbf{3}$ & $\mathbf{4}$ & $\mathbf{5}$ \\
\hline Ações & 16 & 50 & 14 & 5 & 6 \\
Variância Intraclasse & 6,61 & 23,78 & 56,56 & 37,6 & 19,06 \\
\hline
\end{tabular}

Ao verificar a relação Risco versus Retorno dentro dos grupos que foram formulados, pôde-se observar uma notável diferenciação entre eles. Por meio da Figura 3, foi realizada a caracterização dos clusters, valendo-se de sua característica entre risco $x$ retorno, criando-se, assim, uma carteira hipotética a ser sugerida ao potencial investidor.

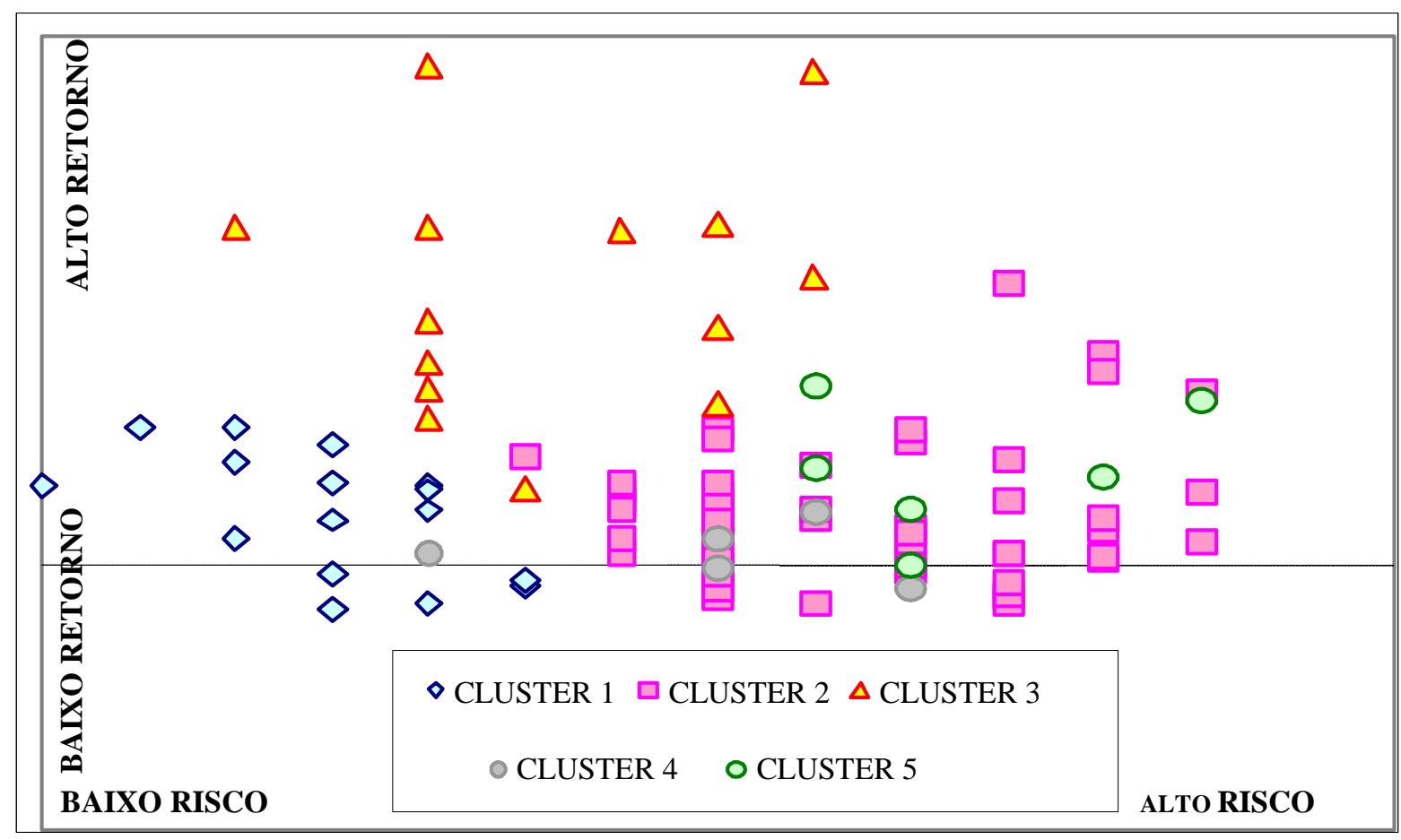

Figura 3 - Relação entre risco $x$.retorno dentro das carteiras 
A carteira 1 é avaliada como BAIXO RISCO e BAIXO RETORNO, que é uma carteira indicada para investidores com pouca tolerância a riscos. A carteira 2 é caracterizada em função de possuir um ALTO RISCO e RETORNO MODERADO, ou seja, são ações que aparentemente não compensam o risco incorrido. A carteira 3 apresenta BAIXO RISCO e ALTO RETORNO, mostrando-se como a melhor opção de investimento entre os grupos formados. A carteira 4 exibe RISCO MODERADO e BAIXO RETORNO, indicando ações que não compensam a exposição ao risco. Finalmente, a carteira 5 tem ALTO RISCO e ALTO RETORNO, sendo indicada apenas para investidores com boa tolerância às oscilações do mercado.

A Tabela 5 apresenta uma comparação entre os diversos indicadores investigados dentro das carteiras sugeridas ao potencial investidor em função do total pesquisado. Estes dados corroboram a caracterização realizada para cada uma das carteiras sugeridas. Por essa análise, as carteiras ou grupos com ações que poderiam ser recomendadas para investimento são o 1,3 e 5, respectivamente.

Tabela 5 - Caracterização das carteiras no período 1998 - 2002

\begin{tabular}{|c|c|c|c|c|c|c|c|}
\hline \multicolumn{8}{|c|}{ CARTEIRAS } \\
\hline Índices & & 1 & 2 & 3 & 4 & 5 & Total \\
\hline AÇÕES & $\mathrm{Q}$ & 16 & 50 & 14 & 5 & 6 & 91 \\
\hline VALORZ & $\mathrm{M}$ & $95,38 \%$ & $100,24 \%$ & $523,78 \%$ & $23,96 \%$ & $184,90 \%$ & $165,94 \%$ \\
\hline BETA & M & 0,30 & 0,85 & 0,53 & 0,70 & 0,95 & 0,70 \\
\hline VOLAT & $\mathrm{M}$ & 0,44 & 0,51 & 0,36 & 0,50 & 0,45 & 0,47 \\
\hline LIQUI & $S$ & $0,43 \%$ & $20,95 \%$ & $6,91 \%$ & $1,31 \%$ & $24,78 \%$ & $54,38 \%$ \\
\hline PTRLQ R\$ & M & $956.553,31$ & $2.797 .225,48$ & $9.569 .560,21$ & 4.937.972,40 & $26.569 .289,50$ & $5.200 .500,76$ \\
\hline PVPA & $\mathrm{M}$ & 2,29 & 3,18 & 8,08 & 35,15 & 6,53 & 5,76 \\
\hline EXGAT & M & 54,49 & 61,42 & 56,02 & 67,34 & 62,72 & 59,78 \\
\hline
\end{tabular}

Nota: Q = Quantidade; $\mathrm{M}=$ Média; $\mathrm{S}=$ Soma

Conforme evidenciado no Quadro 2, têm-se tanto as empresas quanto os respectivos setores das carteiras recomendadas ao potencial investidor.

À luz dos resultados encontrados, percebe-se que a carteira 1, descrita como baixo risco e baixo retorno, apresentou valorização de $95 \%$ nos quatro anos, com um risco bem abaixo da média $\left(\beta_{1}=0,3\right)$. O grupo é constituído de ações de empresas de menor porte e com baixa liquidez na Bolsa de Valores.

A carteira 2, caracterizada por alto risco e retorno moderado, exibiu valorização de $100 \%$ no período; no entanto, o risco $\left(\beta_{2}=0,85\right)$ é $20 \%$ maior que a média. Este grupo, por constituir-se de um número elevado de ações, responde por $21 \%$ da movimentação da Bovespa.

A carteira 3, com baixo risco e alto retorno, ostentou um desempenho muito acima da média $-523 \%$ de valorização - com um risco relativamente baixo $\left(\beta_{3}=0,53\right)$. O cluster possui ações de empresas maiores que os grupos anteriores.

A carteira 4, de risco moderado e baixo retorno, apresentou o pior desempenho dentre os grupos, com valorização de apenas $23,96 \%$ no quadriênio. O risco está compatível com a média $\left(\beta_{4}=0,7\right)$. Os papéis neste agrupamento têm baixa liquidez, mas a proporção do preço das ações pelo valor patrimonial é a maior entre todas as carteiras.

A carteira 5, caracterizada pelo alto risco e alto retorno, obteve $184 \%$ de valorização no período, com risco $\beta_{5}=0,95$. As ações deste agrupamento são de empresas de grande porte e com altíssima liquidez $(24,78 \%)$. 


\begin{tabular}{|c|c|c|c|}
\hline & & EMPRESA & SETOR \\
\hline \multirow{6}{*}{$\begin{array}{c}\mathbf{C} \\
\mathbf{A} \\
\mathbf{R} \\
\mathbf{T} \\
\mathbf{E} \\
\mathbf{I} \\
\mathbf{R} \\
\mathbf{A} \\
\mathbf{5}\end{array}$} & BBDC4 & Bradesco & Bancos \\
\hline & ELET6 & Eletrobras & Energia Elétrica \\
\hline & ITAU4 & Bco Itau & Bancos \\
\hline & PETR3 & Petrobras & $\begin{array}{l}\text { Extração de } \\
\text { petróleo e gás }\end{array}$ \\
\hline & PETR4 & Petrobras & $\begin{array}{l}\text { Extração de } \\
\text { petróleo e gás }\end{array}$ \\
\hline & VALE5 & $\begin{array}{c}\text { Vale Rio } \\
\text { Doce }\end{array}$ & $\begin{array}{c}\text { Mineração de } \\
\text { metais }\end{array}$ \\
\hline
\end{tabular}

\begin{tabular}{|c|c|c|c|}
\hline & & EMPRESA & SETOR \\
\hline $\begin{array}{l}\mathbf{R} \\
\mathbf{T} \\
\mathrm{E} \\
\mathbf{I} \\
\mathbf{R} \\
\mathbf{1}\end{array}$ & AMBV3 & Ambev & Bebidas \\
\hline & BFIT3 & Sudameris & Bancos \\
\hline & BMCT4 & Merc S. Paulo & Bancos \\
\hline & BMTO4 & Brasmotor & Eletrodomésticos \\
\hline & BRGE12 & Alfa Consorcio & Adm. de empresas \\
\hline & BRIV4 & Alfa Invest. & Bancos \\
\hline & DXTG4 & Dixie Toga & Prod. de plásticos \\
\hline & ETER3 & Eternit & Minerais não metálicos \\
\hline & FLCL5 & F Cataguazes & Energia elétrica \\
\hline & MSAN3 & Bunge Brasil & Alimentos \\
\hline & PMAM4 & Paranpanema & Prod. de metal \\
\hline & POMO4 & Marcopolo & Carrocerias e trailers \\
\hline & PRGA4 & Perdigao & Abatedouros \\
\hline & RPAD3 & Alfa Holding & Adm. de empresas \\
\hline & RPAD5 & Alfa Holding & Adm. de empresas \\
\hline & TRFO4 & Trafo & Energia elétrica \\
\hline
\end{tabular}

\begin{tabular}{|c|c|c|c|}
\hline & & EMPRESA & SETOR \\
\hline \multirow{14}{*}{\begin{tabular}{|c}
$\mathbf{C}$ \\
$\mathbf{A}$ \\
$\mathbf{R}$ \\
$\mathbf{T}$ \\
$\mathbf{E}$ \\
$\mathbf{I}$ \\
$\mathbf{R}$ \\
$\mathbf{A}$ \\
$\mathbf{3}$
\end{tabular}} & ALPA4 & Alpargatas & Calçados \\
\hline & ARCE4 & Arcelor BR & Aço \\
\hline & CMET4 & Caemi & $\begin{array}{c}\text { Mineração de } \\
\text { metais }\end{array}$ \\
\hline & CRUZ3 & Souza Cruz & Aço \\
\hline & CSNA3 & Sid Nacional & Aço \\
\hline & EBCO4 & Embraco & $\begin{array}{c}\text { Equip. de } \\
\text { refrigeração }\end{array}$ \\
\hline & EMBR4 & Embraer & Equip. aeroespaciais \\
\hline & FJTA4 & $\begin{array}{l}\text { Forjas } \\
\text { Taurus }\end{array}$ & Prod. de metal \\
\hline & LEVE4 & Metal Leve & Autopeças \\
\hline & OXIT4 & Oxiteno & Química básica \\
\hline & SUZB5 & $\begin{array}{c}\text { Suzano } \\
\text { Papel } \\
\end{array}$ & $\begin{array}{l}\text { Papel, celulose e } \\
\text { papelão }\end{array}$ \\
\hline & UNIP6 & Unipar & Química \\
\hline & VALE3 & $\begin{array}{l}\text { Vale Rio } \\
\text { Doce } \\
\end{array}$ & $\begin{array}{c}\text { Mineração de } \\
\text { metais }\end{array}$ \\
\hline & VCPA4 & $\begin{array}{l}\text { Votorantim } \\
\text { C P } \\
\end{array}$ & $\begin{array}{c}\text { Papel, celulose e } \\
\text { papelão }\end{array}$ \\
\hline
\end{tabular}

Quadro 2 - Composição das carteiras recomendadas

\subsection{Análise do desempenho das carteiras formuladas}

Para validar essa métrica, foi observado se as carteiras constituídas com base nos dados do ano de 2002 possuíam um comportamento em termos de Risco versus Retorno esperado, utilizando-se os dados das mesmas ações, só que para o ano 2006 e verificado a exposição da carteira. Percebeu-se que, dos 91 títulos analisados, 22 ações no total não apresentaram resultado em junho de 2006 , e nove eram constituintes da carteira 1 , dez da carteira 2, duas da carteira 3 e uma da carteira 4.

O cenário conjuntural do mercado financeiro brasileiro foi muito positivo no quadriênio de 2002 a 2006, portanto, o investidor que fez uma aposta em títulos negociados na Bovespa, na média geral, obteve lucros expressivos durante esse período. Esse panorama contribuiu para que, numa análise puramente matemática, no período de 1998 a 2002, ficasse 
muito aquém, principalmente no que tange a variável retorno realizado. A Figura 4 e a Tabela 6 esclarecem numérica e visualmente tal afirmação (apenas para os títulos com informações divulgadas em junho de 2006).

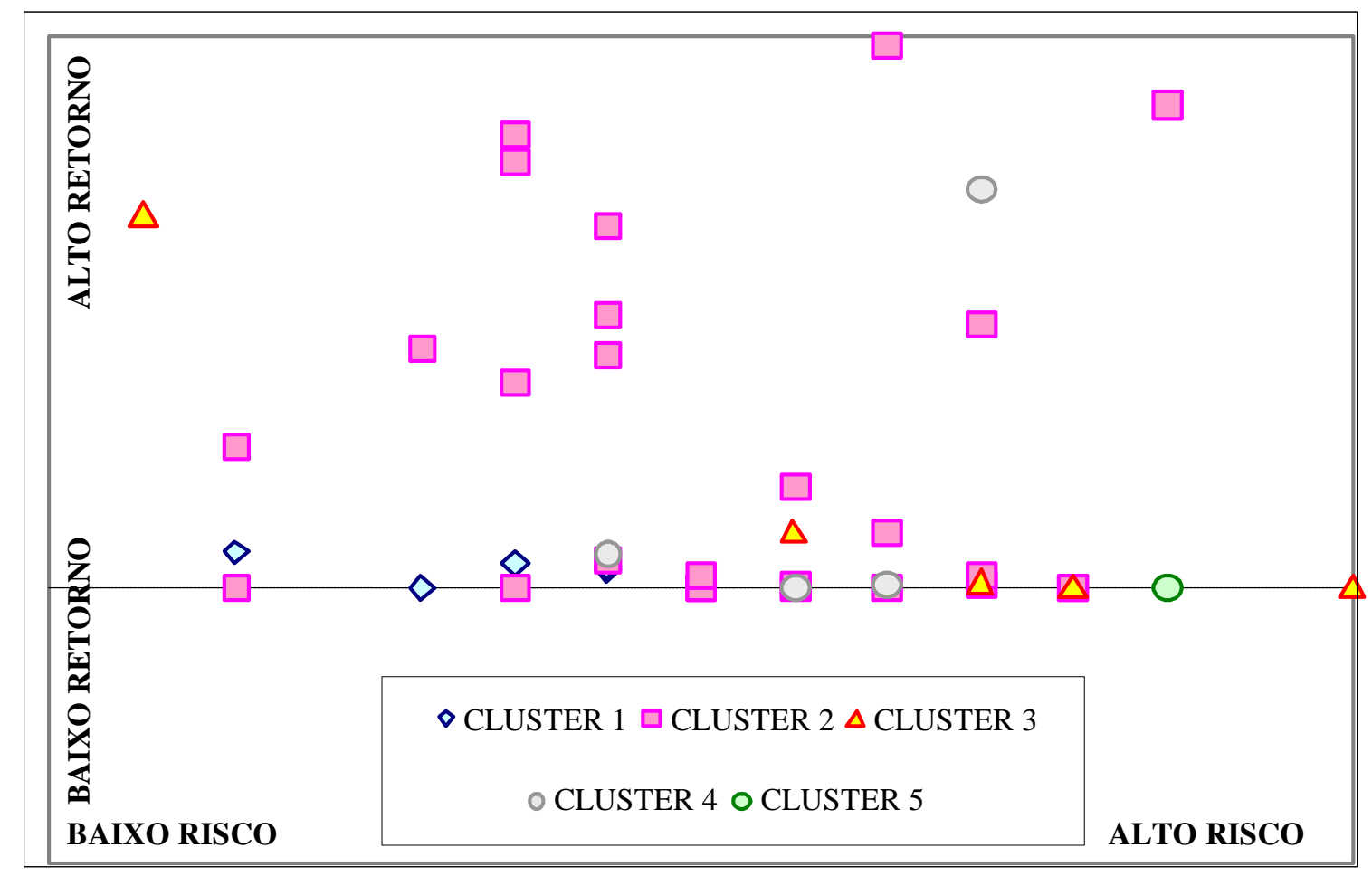

Figura 4 - Comparação do risco versus retorno das carteiras em 30/jun/2006

A Tabela 6 mostra que, no mercado como um todo, verificou-se um aquecimento no período de 2002 a 2006, e a rentabilidade total cresceu exponencialmente. Também é possível identificar que as empresas listadas na Bovespa observaram um crescimento médio bastante relevante no patrimônio, na ordem de $84 \%$. Um índice que não apresentou aumento significativo foi a razão entre preço e valor patrimonial. Aliado a esses indicadores, o risco $(\beta)$ apresentou um crescimento aproximado de $8 \%$, e a volatilidade das ações caiu cerca de $8 \%$.

Tabela 6 - Média da valorização e do risco nos períodos 1998-2002 e 2002-2006 para as carteiras

\begin{tabular}{c|c|c|c|c|c|c|c}
\hline \multicolumn{2}{c|}{} & \multicolumn{2}{c|}{ VALORZ } & \multicolumn{2}{c}{ BETA } & \multicolumn{2}{c}{ LIQUI } \\
\hline CARTEIRA & AÇÕES & $\mathbf{9 8 - 0 2}$ & $\mathbf{0 2}-\mathbf{0 6}$ & $\mathbf{9 8 - 0 2}$ & $\mathbf{0 2}-\mathbf{0 6}$ & $\mathbf{9 8}-\mathbf{0 2}$ & $\mathbf{0 2}-\mathbf{0 6}$ \\
\hline TOTAL & 69 & $187,56 \%$ & $1593,87 \%$ & 0,733 & 0,757 & $47,40 \%$ & $65,50 \%$ \\
$\mathbf{1}$ & 7 & $129,00 \%$ & $1684,73 \%$ & 0,271 & 0,543 & $0,40 \%$ & $0,10 \%$ \\
$\mathbf{2}$ & 40 & $104,02 \%$ & $1221,63 \%$ & 0,848 & 0,758 & $15,60 \%$ & $10,40 \%$ \\
$\mathbf{3}$ & 12 & $557,86 \%$ & $2319,58 \%$ & 0,525 & 0,800 & $5,40 \%$ & $15,90 \%$ \\
$\mathbf{4}$ & 4 & $16,92 \%$ & $196,72 \%$ & 0,700 & 0,825 & $1,20 \%$ & $5,70 \%$ \\
\hline $\mathbf{5}$ & 6 & $184,90 \%$ & $3449,52 \%$ & 0,950 & 0,867 & $24,80 \%$ & $33,40 \%$ \\
\hline
\end{tabular}

Outro fator interessante a ser avaliado refere-se à questão da liquidez total das carteiras. Em junho de 2002, os cinco grupos formados representavam 54\% (47\% sem as ações excluídas) do total da movimentação da Bovespa, enquanto em junho de 2006, mesmo com uma quantidade menor de ações, o percentual total dos agrupamentos situou-se em aproximadamente $66 \%$, demonstrando um aumento de $20 \%$ na representatividade dos títulos selecionados para este estudo. A Tabela 7 traz a descrição das variáveis estudadas para as carteiras hipotéticas sugeridas, no período de 2002 a 2006: 
Carteira 1 - este grupo de ações, indicado como sendo de baixo risco e baixo retorno, mesmo assim obteve, no período 2002-2006, um desempenho acima da média (valorização nominal de 5,7\% superior), com um risco de quase $30 \%$ menor do que a média. Mostrou-se um ótimo investimento, no entanto, como a liquidez na Bolsa de Valores estava muito baixa, representou somente $0,09 \%$ do volume negociado. A proporção entre o preço da ação e o valor patrimonial foi maior do que a média e a volatilidade foi $20 \%$ menor que a média;

Carteira 2 - o investimento na carteira 2 não é recomendado a partir dessa análise a um potencial investidor, haja vista que obteve uma valorização $23 \%$ menor do que a média total dos papéis com um risco igual. Mesmo assim, a liquidez de suas ações representou $10 \%$ do total dos negócios na Bolsa de Valores. Por outro lado, a razão entre o preço da ação e o valor patrimonial situou-se em torno de $8 \%$ menor que a média;

Carteira 3 - as ações formadoras no grupo 3 corresponderam ao esperado, pois obtiveram um retorno $46 \%$ maior do que o valor médio, apesar de ostentarem um risco $6 \%$ maior. A liquidez desses papéis situou-se em $16 \%$ do volume negociado. A razão entre o valor patrimonial e o preço da ação foi o mais alto entre todos os clusters;

Carteira 4 - grupo com o menor percentual de valorização no quadriênio 1998-2002. Esta carteira continuou com um desempenho muito abaixo do valor médio. $\mathrm{O}$ retorno das ações não passou de $197 \%$, quase $90 \%$ a menos de valorização do que a média geral da Bovespa. $\mathrm{O}$ risco beta desta carteira foi um dos mais elevados, mesmo a carteira sendo caracterizada como conservadora $(\beta=0,83)$;

Carteira 5 - este grupo de ações continua sendo muito atraente apesar do seu risco beta $(\beta=$ 0,87 ). Ela alcançou uma rentabilidade de $116 \%$ superior à média. $\mathrm{O}$ volume negociado representou 33,5\% das transações da Bolsa de Valores. Constituiu-se de empresas de grande porte, com um valor por ação em relação ao patrimônio 7\% acima da média. No entanto, a volatilidade dos papéis foi a maior entre os grupos, situando-se em torno de $21 \%$ superior à média geral.

Tabela 7 - Caracterização das carteiras no período 2002-2006

\begin{tabular}{cccccccc}
\hline \multicolumn{7}{c}{ CARTEIRAS } \\
\hline Índices & & $\mathbf{1}$ & $\mathbf{2}$ & $\mathbf{3}$ & $\mathbf{4}$ & $\mathbf{5}$ & Total \\
\hline AÇÕES & $\mathrm{Q}$ & 7 & 40 & 12 & 4 & 6 & 69 \\
VALORZ & $\mathrm{M}$ & $1684,73 \%$ & $1221,63 \%$ & $2319,58 \%$ & $196,72 \%$ & $3449,52 \%$ & $1593,87 \%$ \\
BETA & $\mathrm{M}$ & 0,54 & 0,76 & 0,80 & 0,83 & 0,87 & 0,76 \\
VOLAT & $\mathrm{M}$ & 0,34 & 0,42 & 0,48 & 0,45 & 0,52 & 0,43 \\
LIQUI & $\mathrm{S}$ & $0,09 \%$ & $10,35 \%$ & $15,93 \%$ & $5,65 \%$ & $33,45 \%$ & $65,47 \%$ \\
PTRLQ R\$ & $\mathrm{M}$ & $2.696 .788,00$ & $3.861 .687,11$ & $17.716 .525,33$ & $7.974 .901,33$ & $37.840 .516,25$ & $9.569 .961,72$ \\
PVPA & $\mathrm{M}$ & 14,91 & 11,28 & 17,31 & 1,15 & 13,13 & 12,26 \\
EXGAT & $\mathrm{M}$ & 65,95 & 59,23 & 48,58 & 73,47 & 59,98 & 58,73 \\
\hline
\end{tabular}

Nota: $Q=$ Quantidade; $M=$ Média $;$ = Soma.

Os resultados apresentados anteriormente demonstram que a estatística multivariada de cluster analisys pode ser utilizada na alocação de títulos em carteiras ou portfolios. A formulação de grupos a partir de informações do período 1998 a 2002 leva à construção de carteiras hipotéticas sugeridas que apresentaram desempenho dentro do esperado no quadriênio 2002 a 2006. No entanto, é válido salientar que as mudanças no padrão do mercado acionário podem causar distorções perigosas nos resultados da análise, portanto, a análise conjuntural aliada aos estudos matemáticos como pesquisa operacional e (ou) econométricos é indispensável à construção de boas carteiras de investimento. 


\section{CONSIDERAÇÕES FINAIS}

Este estudo procurou, por meio da técnica de análise de agrupamentos ou cluster analysis, formular grupos de ações com características semelhantes para constituir carteiras hipotéticas sugeridas a um investidor, também hipotético, da Bolsa de Valores de São Paulo. O período analisado foi o quadriênio de 1998 a 2002, para a construção dos clusters, e de 2002 a 2006, para a avaliação do comportamento desses clusters.

Os resultados mostraram que as carteiras formadas mantiveram um desempenho percentual em conformidade com o esperado, confirmando o fato de que o ferramental estatístico é um bom aliado no desenvolvimento de grupos de títulos. No entanto, o mercado acionário brasileiro obteve, no período de 2002 a 2006, resultados muito expressivos, portanto, as taxas de retorno esperadas foram muito inferiores àquelas realizadas.

É necessário ressaltar que as mudanças ocorridas no cenário econômico brasileiro e mundial reforçam a necessidade de análises macroeconômicas específicas para fundamentar qualquer tentativa de antecipação de movimentação dos títulos do mercado acionário.

A pesquisa mostrou a eficaz utilização dessa técnica estatística na construção de carteiras de ativos. Não foi realizada a previsão de retornos, pois entende-se que não é possível analisar o mercado financeiro apenas com base em dados históricos, sem se considerar também a conjuntura econômica do país ou mesmo do mundo. Também não foi focada a questão da alocação ótima de capital dentro de cada uma das carteiras, sendo este assunto de relevante interesse para o desenvolvimento de trabalhos futuros.

Estudos interessantes nessa área poderão ser realizados na formulação de carteiras, fazendo-se previsões de risco versus retorno a partir da construção de cenários prospectivos de acordo com as projeções econômicas traçadas por analistas, governos ou entidades de mercado.

\section{REFERÊNCIAS}

BARROSO, L. P.; ARTES, R. Minicurso de análise multivariada. In: REUNIÃO DA RBRAS, 48., SEAGRO, 10., 2003. Lavras. Anais... Lavras: Departamento de Ciências Exatas, Universidade Federal de Lavras, 2003.

BLACK, F.; LITTERMAN, R. Asset allocation: combining investors views with market equilibrium. Fixed Income Research, Goldman, Sachs \& Company, September. 1990.

BOLSA DE VALORES DE SÃO PAULO (BOVESPA). Disponível em: <http://www.bovespa.com.br/Principal.asp >. Acesso em: 15 jan. 2007.

CIA, J. C.; GUARITA, C.; PEDOTE, C. Mercado acionário brasileiro: um estudo do comportamento das ações com o uso de cluster analysis. In: ENANPAD, 23., 1999, Foz do Iguaçu. Anais... Rio de Janeiro: ANPAD, 1999. CD ROM.

COSTA, O. L. V.; ASSUNÇÃO, H. G. V. Análise de risco e retorno em investimentos financeiros. Barueri: Manole, 2005.

COOPER, I.; KAPLANIS, E. What explains the home bias in portfolio investment. Working Paper, London Business School. 1991.

DAMODARAN, A. Finanças corporativas: teoria e prática. Porto Alegre: Bookman, 2004.

DILLON, W. R.; GOLDSTEIN, M. Multivariate analysis: methods and applications. New York: John Wiley \& Sons, 1984.

DIMSON, E. M.; MUSSAVIAN, M. Three centuries of asset pricing. Journal of Banking and Finance, v. 23, p. 1745-1769, 1999. http://dx.doi.org/10.1016/S0378-4266(99)00037-0 
ELTON, E. J. et al. Moderna teoria de carteiras e análise de investimentos. 6. ed. São Paulo: Atlas, 2004.

ELTON, E. J.; MARTIN J. G.; PADBERG, M. W. Simple rules for optimal portfolio selection: the Multi Group case. Journal of Financial and Quantitative Analysis, n. 32, p.329-345, 1977. http://dx.doi.org/10.2307/2330538

FRANCIS, J. C. Investments: analysis and management. 5. ed. New York, McGraw-Hill, 2001.

FRENCH, K.; POTERBA, J. M. Investor diversification and international equity markets, The American Economic Review, v. 81, p. 222-226, 1991. http://www.jstor.org/stable/2006858

GIL, A. Como elaborar projetos de pesquisa. 4. ed. São Paulo: Atlas, 2002.

GITMAN, L. J.; JOEHNK, M. D. Princípios de investimentos. São Paulo: Pearson Addison Wesley, 2005.

GRUBEL, H. G. Internationally diversified portfolios: welfare gains and capital flows, The American Economic Review, n. 58, p. 1299-1314, Dec. 1968.

KRITZMAN, M. Risco e utilidade: o básico. In: BERNSTEIN, P. L.; DAMODARAN, A. Administração de investimentos. Porto Alegre: Bookman, 2000. p. 37-81.

LEVINE, D. M. et al. Estatística: teoria e aplicações usando o Microsoft Excel em português. Rio de Janeiro: LTC, 2005.

LINTZ, A.; RENYI, L. Análise de diversificação de carteiras compostas por ações pertencentes ao índice Bovespa: uma análise a partir do modelo de Sharpe. Caderno de Pesquisas em Administração, São Paulo, v. 11, n. 7, p. 12, 2. trim./1998.

MALHOTRA, N. K. Pesquisa de marketing: uma orientação aplicada. Porto Alegre: Bookman, 2004.

MARKOWITZ, H. Portfolio selection. Journal of Finance, v. 7 p. 77-91, 1952. http://dx.doi.org/10.2307/2975974

RIBEIRO, C. O.; FERREIRA, L. A. S. Uma contribuição ao problema de composição de carteiras de mínimo valor em risco. Gestão \& Produção, v. 12, n. 2, p. 295-304, 2005.

TOSTA DE SÁ, Geraldo. Administração de investimentos: teoria de carteiras e gerenciamento de riscos. Rio de Janeiro: Qualitymark, 1999.

VINCE, R. Cálculo e análise de riscos no mercado financeiro. São Paulo: Makron Books, 1999. 